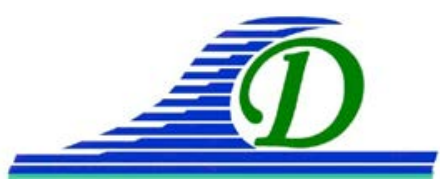

XIII ${ }^{\text {èmes }}$ Journées Nationales Génie Côtier - Génie Civil

Dunkerque, 2-4 juillet 2014

DOI:10.5150/jngcgc.2014.055 C Editions Paralia CFL

disponible en ligne - http://www.paralia.fr - available online

\title{
Evolution hydrosédimentaire récente de l'estuaire de la Gironde. Apport d'un modèle de transport
}

\section{Aldo SOTTOLICHIO ${ }^{1}$, Vincent HANQUIEZ ${ }^{1}$, Barend VAN MAANEN ${ }^{1}$, Joselyn ARRIAGADA ${ }^{1}$, Isabel JALON ROJAS ${ }^{1}$, Sabine SCHMIDT ${ }^{2}$, Florent BIRRIEN ${ }^{1}$}

\author{
1. Université de Bordeaux, Laboratoire EPOC, UMR 5805, F-33 Pessac, France. \\ aldo.sottolichio@u-bordeaux.fr \\ 2. CNRS, EPOC, UMR 5805, F-33400 Talence, France.
}

\section{Résumé :}

La modélisation 3D de la turbidité en estuaire et les applications à des systèmes réels se sont beaucoup développées ces dernières années. Toutefois, si la validation du calcul hydrodynamique 3D est relativement aisée, la calibration sédimentaire est beaucoup plus compliquée. Pour ces raisons, encore peu de modélisations ont tenté de quantifier les bilans sédimentaires basés sur la dynamique saisonnière du bouchon vaseux. Dans cette communication, nous présentons une modélisation de l'estuaire de la Gironde, avec en particulier un essai de validation du bouchon vaseux grâce aux mesures et observations spatiales récentes et disponibles. Nous montrons que les bilans sédimentaires sont très sensibles au paramétrage de la vitesse de chute, qui peut être ajustée par l'examen de la stabilité de la turbidité à moyen terme. Enfin, nous discutons de l'évolution hydrosédimentaire de la Gironde à plus long terme par l'application du modèle dans deux configurations bathymétriques récentes des fleuves. Leur comparaison montre que des effets anthropiques locaux ont pu contribuer à une remontée du bouchon vaseux dans les fleuves à l'échelle de 50 ans, indépendamment de tout effet climatique global ou régional.

Mots-clés : Dynamique sédimentaire, Estuaire de la Gironde, Modèle, Bouchon vaseux.

\section{Introduction}

La modélisation 3D de la turbidité en estuaire s’est beaucoup développée ces dernières années, et les applications à des systèmes réels ont permis de décrire le bouchon vaseux ou de quantifier des flux en suspension dans plusieurs environnements (PARK et al., 2008 ; SOUZA et al., 2012). Le calcul 3D est indispensable car les modèles intégrés sur la verticale $(2 \mathrm{DH})$ ne peuvent pas restituer les gradients verticaux ni les stratifications de densité. Toutefois, si la validation du calcul hydrodynamique (courants moyens) est relativement aisée, la calibration sédimentaire est plus compliquée car la concentration est contrôlée par plusieurs paramètres dépendant des propriétés des sédiments. Pour ces raisons, encore peu de modélisations ont tenté de quantifier les bilans sédimentaires saisonniers. 
Le modèle SiAM (BRENON \& LE HIR, 1999), a été implanté dans l'estuaire de la Gironde pour décrire le bouchon vaseux en 2DH puis en 3D (SOTTOLICHIO et al., 2001). Des simulations académiques avaient démontré le rôle prépondérant de l'asymétrie de l'onde de marée dans l'existence du bouchon vaseux, et l'effet secondaire des gradients de densité pour maintenir une masse stable de sédiments en suspension dans l'estuaire. Toutefois, la validation du modèle avait été très sommaire, avec un paramétrage sédimentaire simplifié (une classe de particules avec $w_{s}$ constante). Dans cette communication, nous présentons un essai de validation du bouchon vaseux par des mesures et observations satellitales récentes. L'objectif est de discuter la sensibilité du modèle au paramétrage du comportement sédimentaire, en particulier la vitesse de chute. Enfin, nous discutons de l'évolution hydrosédimentaire de la Gironde à plus long terme par l'application du modèle à deux configurations bathymétriques récentes des fleuves, séparées de 40 ans environ.

\section{Description du modèle SIAM}

Le modèle hydrodynamique résout les équations de Navier Stokes avec une condition de surface libre, simplifiées par l'hypothèse hydrostatique et l'approximation de Boussinesq. Les détails des équations et du schéma numérique sont dans BRENON et LE HIR (1999), et dans BENAOUDA (2008). Le modèle de transport résout une équation d'advection-dispersion avec des termes empiriques d'échange de matière avec le fond, représentatifs du dépôt et de l'érosion. Le flux de dépôt s'écrit selon la formule de Krone (1962), qui dépend de la probabilité de dépôt des particules :

$D=w_{s} C\left(1-\frac{\tau}{\tau_{c d}}\right)$

où $w_{s}$ est la vitesse de chute, $C$ la concentration, $\tau$ la contrainte de cisaillement au fond et $\tau_{c d}$ la contrainte critique de dépôt (pas de dépôt si $\tau>\tau_{c d}$ ). Le flux d'érosion est calculé par la formule de Partheniades (1965), qui dépend de l'excès de cisaillement :

$E=E_{0}\left(\frac{\tau}{\tau_{c e}}-1\right)$

où $E_{0}$ est la constante d'érosion et $\tau_{c e}$ la contrainte critique d'érosion ( $E=0$ pour $\tau<\tau_{c e}$ ). L'érodabilité dépend de la consolidation, gérée par un modèle de tassement 1DV multicouches. Ce modèle résout une équation de conservation de masse, où la sédimentation dépend uniquement de la porosité locale (BRENON \& LE HIR, 1999)

\section{Configuration du modèle de la Gironde}

La grille de calcul couvre l'ensemble de l'estuaire depuis la limite amont de propagation de la marée dans les fleuves (figure 1) et intègre une partie du plateau continental du Golfe de Gascogne. 


\section{XIII ${ }^{\text {ìmes }}$ Journées Nationales Génie Côtier - Génie Civil \\ Dunkerque, 2-4 juillet 2014}
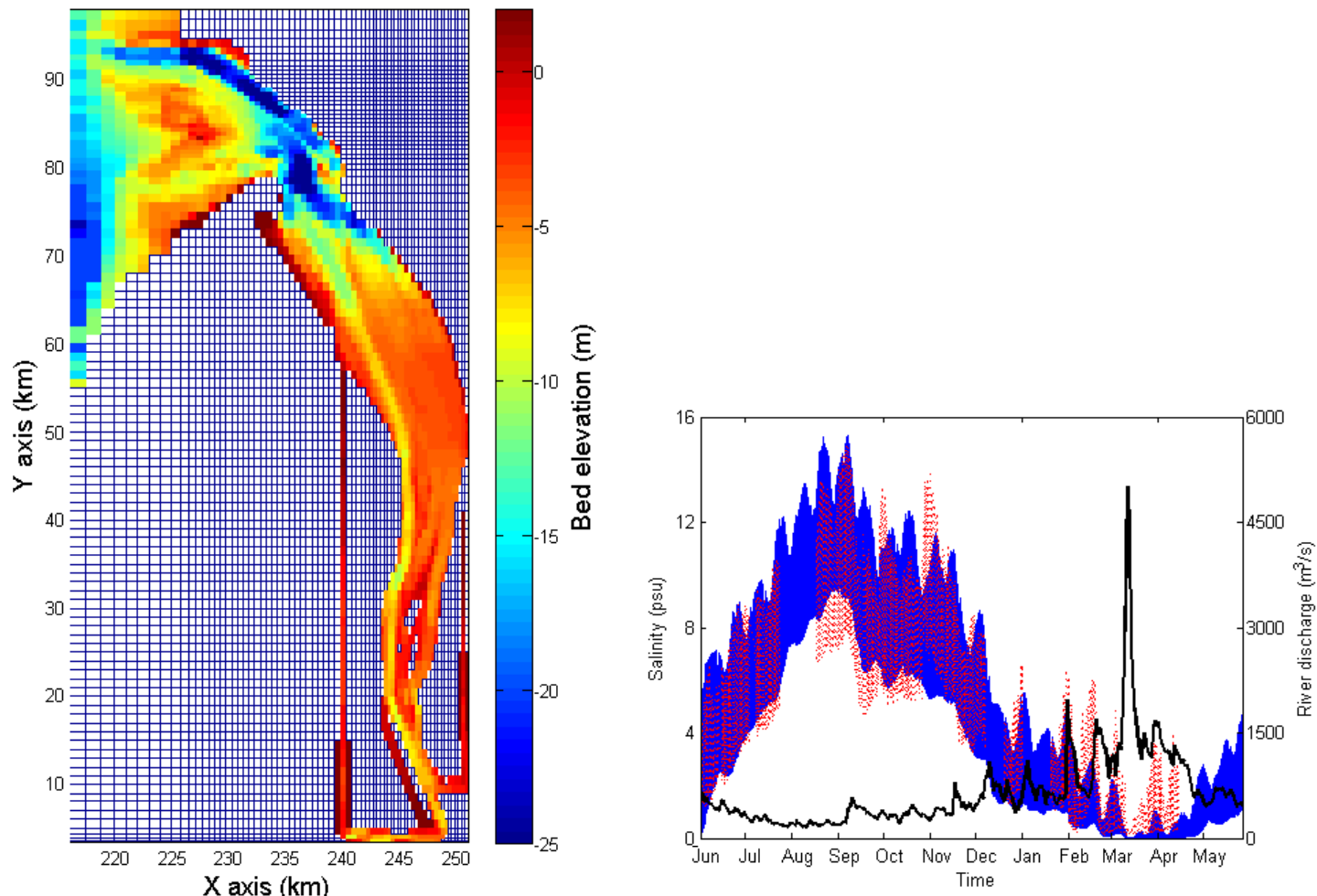

Figure 1. Gauche : Bathymétrie et maillage du modèle SiAM-Gironde. Droite :

Salinités de surface à Pauillac en 2005-2006 (axe de gauche). En rouge: mesures

MAGEST. En bleu : modèle. En noir : débit fluvial Gironde (axe de droite).

La grille est irrégulière, avec une résolution raffinée dans l'estuaire et dans l'axe du chenal (200 m $\times 1 \mathrm{~km})$, et une résolution plus large (>10 km) au-dessus du plateau. La colonne d'eau est divisée en 20 couches d'épaisseur fixe (2 m dans l'estuaire), progressivement croissantes vers le large. Le long de la frontière marine, une marée réelle est imposée par le calcul de 21 composantes harmoniques. Les débits des fleuves Garonne et Dordogne sont introduits quotidiennement à la frontière amont du modèle. Le modèle hydrodynamique a été calibré par ajustement des longueurs de rugosité $z_{0}$, et a été validé en hauteur de marée et vitesses de courant (BENAOUDA, 2008). Une comparaison avec des données in situ plus récentes (mesures continues du réseau MAGEST, ETCHEBER et al., 2011) a permis une validation du modèle en salinité à l'échelle saisonnière, comme par exemple au niveau de Pauillac (figure 1).

\section{Paramétrage du modèle sédimentaire}

\subsection{Paramètres d'érosion et de dépôt}

Le modèle de transport considère deux classes de particules, où la vitesse de chute $w_{s}$ varie en fonction de la concentration, et tient compte implicitement de la floculation et de l'entravement (LE HIR et al., 2001). La constante d'érosion est fixée égale à 


\section{Thème 2 - Dynamique sédimentaire}

$2 \times 10^{-3} \mathrm{~kg} \mathrm{~m}^{-2} \mathrm{~s}^{-1}$ et la tension critique d'érosion $\tau_{c e}$ est fonction de la densité du sédiment superficiel selon une loi puissance (BRENON \& LE HIR, 1999). La densité du sédiment est calculée par le modèle de consolidation. Pour le flux de dépôt, $\tau_{c d}$ est fixée à une valeur élevée $\left(10 \mathrm{~N} \mathrm{~m}^{-2}\right)$, afin de permettre le dépôt à tout moment du cycle de marée.

\subsection{Ajustement de la vitesse de chute}

Les simulations réalisées ont montré que le modèle de transport est sensible à $w_{s}$. Dans les simulations précédentes (SOTTOLICHIO et al., 2001), $w_{s}$ était constante et égale à $1 \mathrm{~mm} \mathrm{~s}^{-1}$. Cette valeur représentait la classe des flocs, mais n'était corroborée par aucune mesure de $w_{s}$ in situ spécifique au bouchon vaseux de la Gironde. Ce type de mesure est d'ailleurs très difficile à réaliser en milieu très turbide. Dans les simulations les plus récentes, nous avons cherché des valeurs optimales de $w_{s}$ par comparaison avec les mesures de turbidité disponibles. Sur la figure 2, les résultats du modèle sur 1 an sont comparés aux mesures MAGEST de turbidité à Pauillac, dans l'estuaire central. Dans le cas $\mathrm{A}, w_{s}$ est choisie relativement faible, ce qui favorise de fortes concentrations en surface, plus proches de ce qu'indiquent les mesures. Cependant, on observe après quelques mois une diminution rapide de la concentration sur le moyen terme, ce qui indique une expulsion excessive du bouchon vaseux vers l'océan. Dans le cas B, la vitesse de chute "optimisée" est plus élevée ; cela se traduit par des concentrations plus faibles en surface. Cependant, la concentration reste stable tout au long du calcul, suggérant une stabilité du bouchon vaseux sur le moyen terme. Malgré la bonne dynamique temporelle de la concentration dans le modèle, il existe des différences importantes avec les mesures. Celles-ci sont réalisées près du bord, ce qui tend à surestimer la concentration de surface. Le modèle, au contaire, a tendance à sousestimer et à lisser la concentration du fait d'une emprise de la maille qui intègre un volume d'eau plus important.

La stabilité du bouchon vaseux dans l'ensemble de l'estuaire peut être vérifiée sur le moyen terme au travers des bilans sédimentaires. La figure 3 montre les bilans pour des simulations de 1 an, avec les deux paramétrages de $w_{s}$ testés dans la figure 2. Pour des vitesses de chute faibles (figures 2 gauche), la quantité totale de MES dans l'estuaire diminue considérablement. Le flux de MES à l'océan atteint $4 \times 10^{6}$ t, ce qui est une valeur excessive d'après l'état des connaissances, qui estime ces flux à des valeurs ne dépassant pas 1 million de tonnes par an (BENAOUDA, 2008).

Dans le cas de la vitesse de chute optimisée (figure 2 droite), la masse totale en suspension diminue modérément, et la vase déposée est plus importante. Le flux de MES exporté à l'océan est nettement inférieur dans les premier mois, et augmente seulement au moment de la crue fluviale du mois de mars, pour un total de $700000 \mathrm{t}$. 

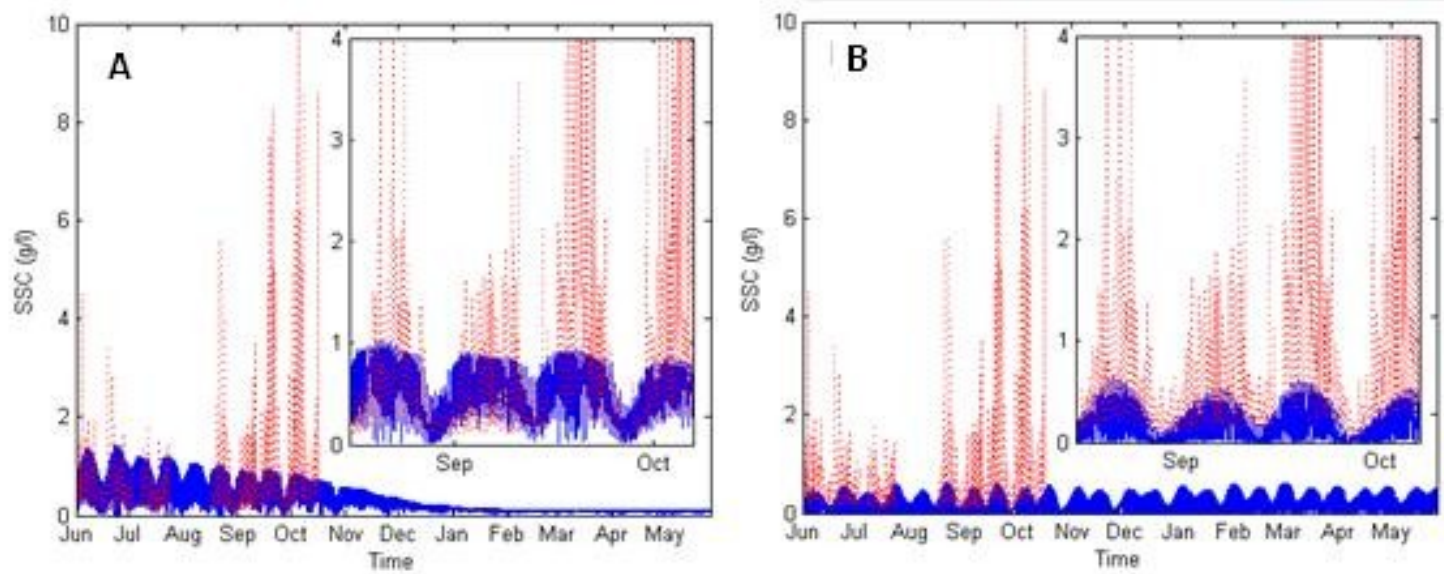

Figure 2. Comparaison des MES calculées (courbes continues bleues) et mesurées (pointillés rouges) à Pauillac en surface. A) ws "réduite" B) ws "optimisée". Le cadre à droite est un zoom sur les mois de septembre et octobre.
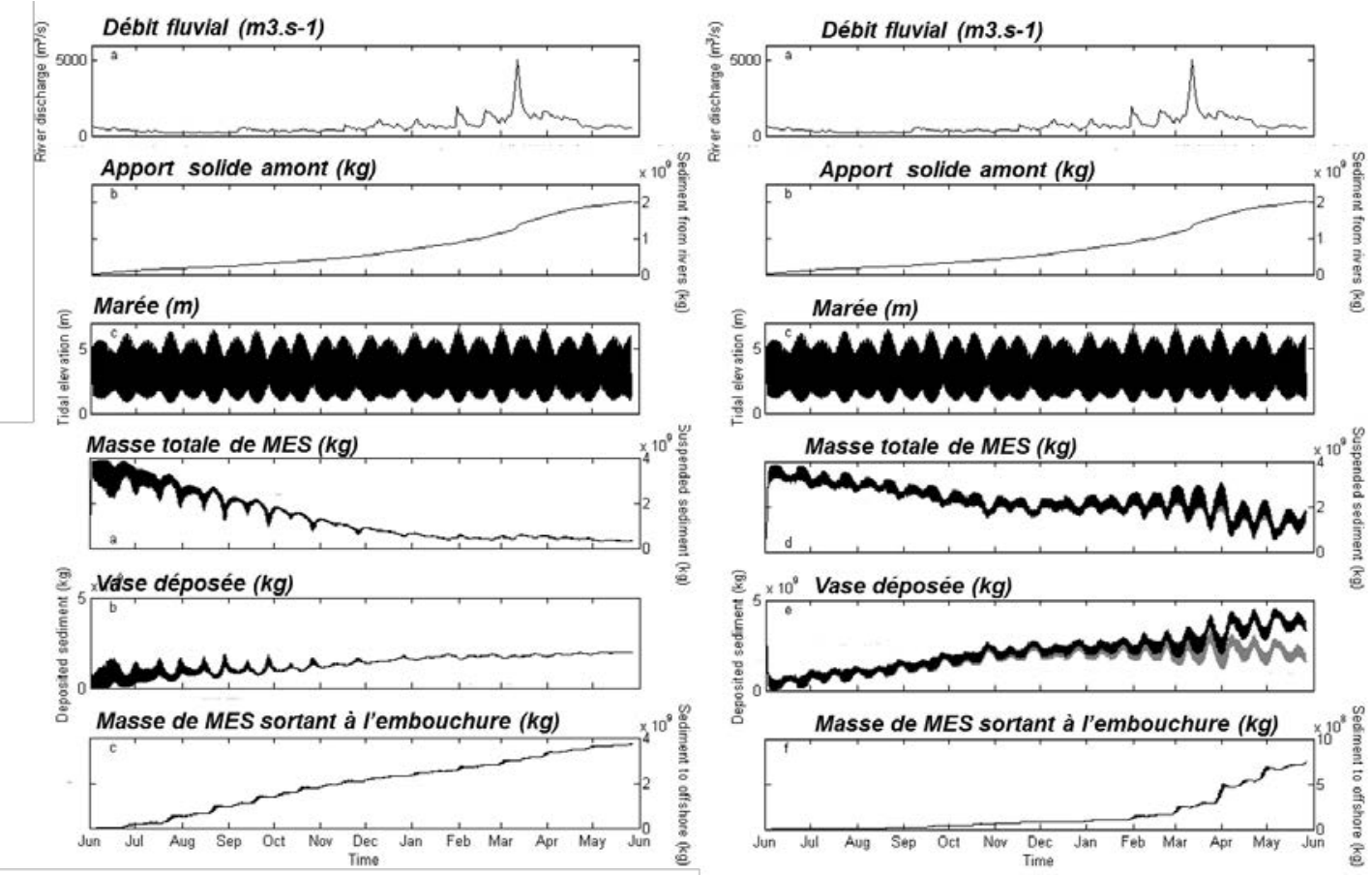

Figure 3. Bilan sédimentaire calculé dans l'estuaire de la Gironde sur 12 mois. A gauche: calcul avec vitesse de chute réduite. A droite : vitesse de chute optimisée.

\subsection{Bouchon vaseux simulé}

Le modèle, avec un parmétrage optimisé, a été comparé avec des images satellites MODIS récentes couvrant différentes situations hydrologiques (DOXARAN et al., 2009, figure 4). La correspondance entre le bouchon vaseux simulé et les images satellites est très satisfaisante, y compris dans la représentation d'un bouchon vaseux secondaire en période de fort débit fluvial. Ceci souligne qualitativement l'influence de 
l'hydrodynamique dans la position du bouchon vaseux, ainsi que la bonne performance de la calibration sédimentaire.
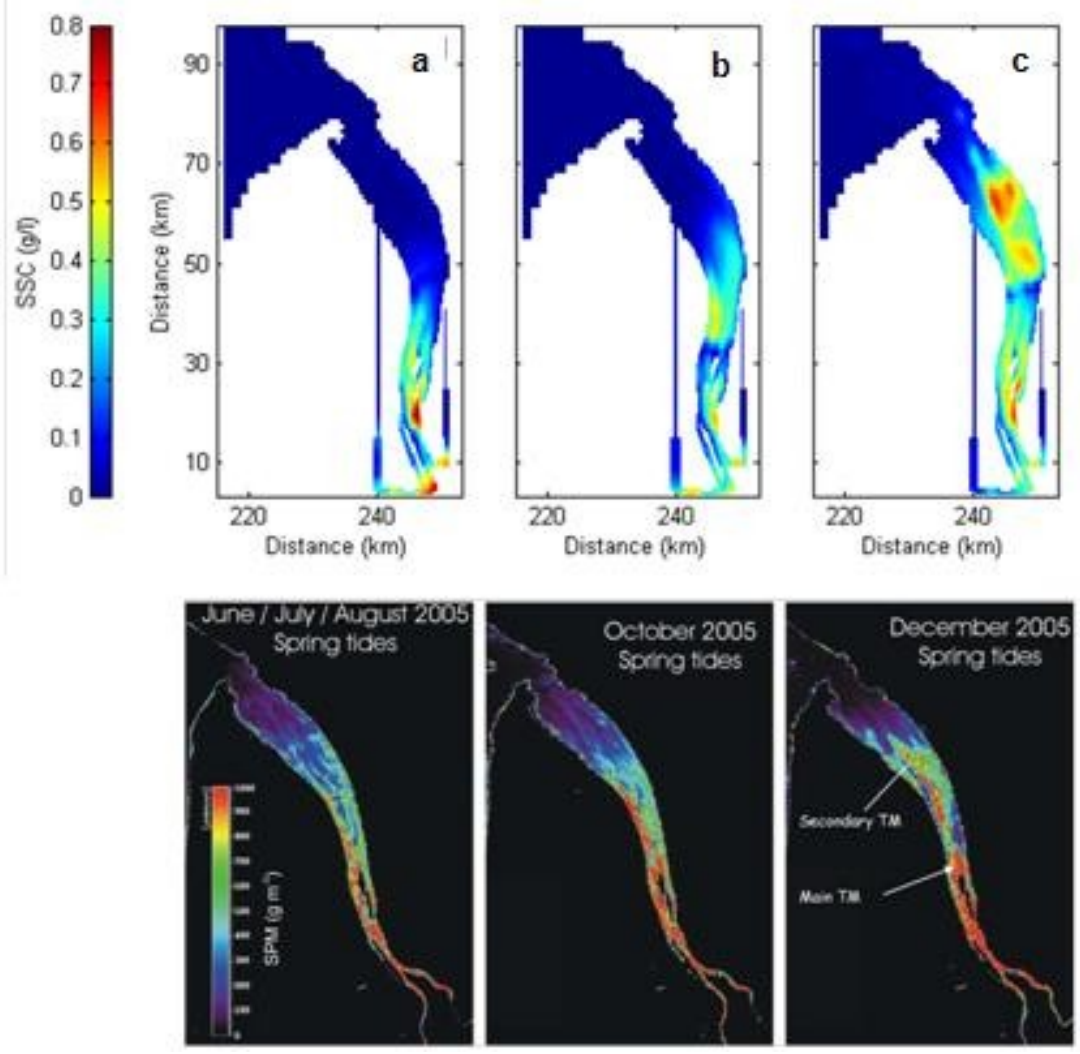

Figure 4. En haut : bouchon vaseux dans l'estuaire de la Gironde pour des débits fluviaux faibles, modérés et forts (concentration en $\mathrm{g}^{-1}$ ). En bas : cartes par inversion

du satellite MODIS pour des conditions de débit similaires, par DOXARAN et al. (2009). Concentrations exprimées en $\mathrm{kg} \mathrm{m}^{-3}$.

\section{Evolution pluridécennale}

L'estuaire fluvial a subi de profonds changements morphologiques au $\mathrm{XX}^{\text {ème }}$ siècle. Entre 1959 et 2002, années des deux dernières couvertures bathymétriques disponibles, la Garonne a subi une érosion généralisée. Les causes principales sont les travaux d'aménagement et, surtout, l'extraction massive de granulats jusqu'au milieu des années 60 en amont de Bordeaux. Le modèle SIAM-3D a été utilisé pour simuler, par des scénarios académiques, l'effet de ce changement bathymétrique dans les fleuves sur la dynamique du bouchon vaseux.

Nous avons utilisé les bathymétries fluviales de 1959 et de 2002, et le modèle a fait l'objet d'une validation hydrodynamique pour chacune des deux situations. La bathymétrie de l'estuaire aval a été maintenue constante, étant donné que la morphologie y a peu changé. Enfin, les simulations ont été effectuées avec des 


\section{XIII $I^{\text {èmes }}$ Journées Nationales Génie Côtier - Génie Civil \\ Dunkerque, 2-4 juillet 2014}

conditions hydrologiques et un paramétrage sédimentaire identiques, afin d'évaluer l'effet unique de la bathymétrie des fleuves.

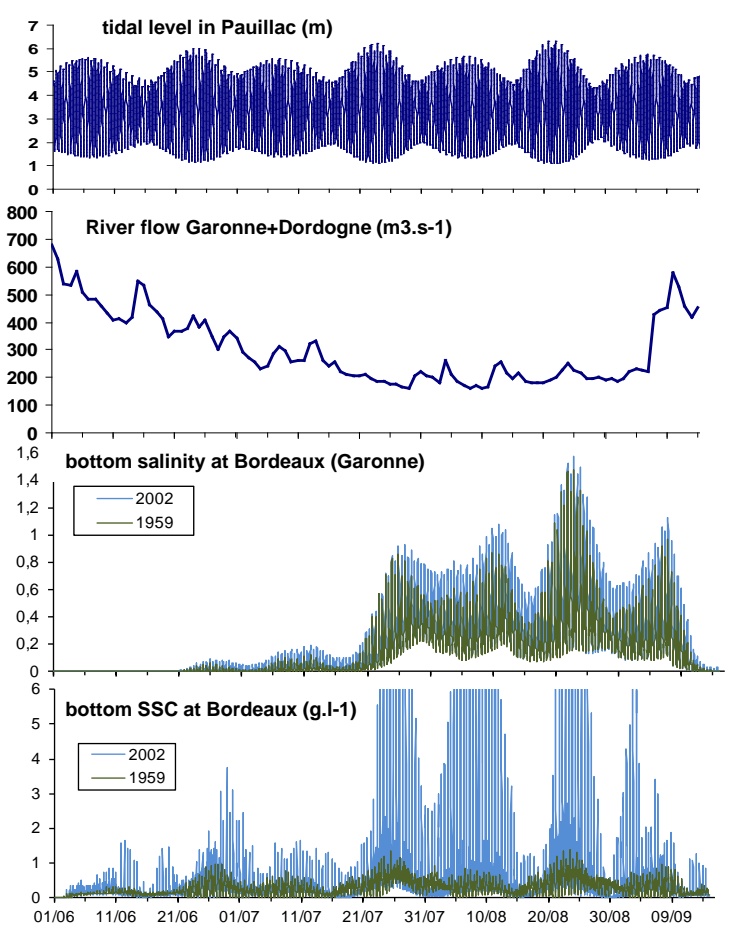

Figure 5. Simulation de la salinité au fond et des MES au fond sur la Garonne, à hauteur de Bordeaux, pour deux bathymétries différentes (1959 et 2002).

Les résultats montrent que l'intrusion saline est peu différente en 2002, mais surtout, que les concentrations sont très supérieures en 2002 par rapport à 1959 (figure 5). De plus, au cours de l'établissement du régime d'étiage, les flux en suspension dirigés vers l'amont dans les jonctions Gironde-Garonne-Dordogne sont plus élevés en 2002 qu'en 1959. Ceci tend à démontrer qu'une remontée effective du bouchon vaseux en étiage s'est produite sur 40 ans, par le seul fait de l'évolution de la topographie fluviale, qui a modifié la propagation de la marée et, en conséquence le transport des sédiments. D'autres travaux ont montré une "marinisation" de l'estuaire aval sur les trente dernières années (CHAALALI et al., 2013), qui serait attribuée à un effet climatique global ou régional. Nos résultats, très préliminaires, suggèrent qu'il peut exister, au moins, une superposition de ces effets globaux avec des effets locaux anthropiques, dûs aux modifications du lit de la Garonne et de la Dordogne.

\section{Conclusions}

Même si le paramétrage de la vitesse de chute est encore très perfectible, le travail de comparaison aux données in situ a permis d'affiner et améliorer de manière significative la qualité des résultats du modèle, et de simuler un bouchon vaseux réaliste, qui se 
maintient dans l'estuaire à moyen terme. Son exploitation dans un exercice de comparaison bathymétrique suggère des changements hydrosédimentaires importants dans l'estuaire. Il est prévu de les étudier plus en détail afin de mieux préciser l'effet des transformations locales par rapport aux différents forçages globaux identifiés.

\section{Références bibliographiques}

BENAOUDA A. (2008). Dynamique saisonnière des sédiments en suspension dans l'estuaire de la Gironde : modélisation opérationnelle de la réponse aux forçages hydrodynamiques. Thèse de l’Université Bordeaux 1, n 3618, 292 p.

BRENON I., LE HIR P. (1999). Modelling the turbidity maximum in the Seine estuary (France): Identification of formation processes. Estuarine, Coastal and Shelf Science, Vol. 49, pp 525-544. http://dx.doi.org/10.1006/ecss.1999.0514

CHAALALI A., CHEVILLOT X., BEAUGRAND G., DAVID V., LUCZAK C., BOET P., SOTTOLICHIO A., SAUTOUR B. (2013). Changes in the distribution of copepods in the Gironde estuary: a warming and marinisation consequence? Estuarine, Coastal and Shelf Science, Vol. 134, pp 150-161. http://dx.doi.org/10.1016/j.ecss.2012.12.004

DOXARAN D., FROIDEFOND J.M., CASTAING P., BABIN M. (2009). Dynamics of the turbidity maximum zone in a macrotidal estuary (the Gironde, France): Observations from field and MODIS satellite data. Estuarine, Coastal and Shelf Science, Vol. 81, pp 321-332. http://dx.doi.org/10.1016/j.ecss.2008.11.013

ETCHEBER H., SCHMIDT S., SOTTOLICHIO A., MANEUX E., CHABAUD G., ESCALIER J.M., WENNEKES H., DERRIENNIC H., SCHMELTZ M., QUEMENER L., REPECAUD M., WOERTHER P., CASTAING P. (2011). Monitoring water quality in estuarine environments: Lessons from the MAGEST monitoring programme in the Gironde fluvial-estuarine system. Hydrology and Earth System Sciences, Vol. 15, pp 831-840. http://dx.doi.org/10.5194/hess-15-831-2011

LE HIR P., BASSOULLET P., JESTIN H. (2001). Application of the continuous modelling concept to simulate high-concentration suspended sediment in a macrotidal estuary. In: McAnally, W.H., Mehta, A.J. (Eds.), Coastal and Estuarine Fine Sediments Processes. Elsevier, Amsterdam, pp 229-247.

SOTTOLICHIO, A., P. LE HIR, P. CASTAING (2001). Modeling mechanisms for the turbidity maximum stability in the Gironde estuary, France, in: W.H. McAnally, A.J. Mehta (Eds.), Coastal and Estuarine Fine Sediment Processes, Elsevier, Amsterdam, pp 373-386.

SOUZA A.J., AMOUDRY L., BOLANOS R. (2012). Modelling sediment transport in a macrotidal, funnel shaped bichannel estuary. The Physics of Estuaries and Coastal Seas (PECS) Symposium. August 12-16 2012, New York City (US).

PARK K., WANG H.V., KIM S.-C., OH J.-H. (2008). A model study of the estuarine turbidity maximum along the main channel of the upper Chesapeake Bay, Estuaries and Coasts, Vol. 31, pp 115-133. http://dx.doi.org/10.1007/s12237-007-9013-8 